\title{
ESR of $\mathrm{Gd}^{3+}$ and $\mathrm{Er}^{3+}$ in $\operatorname{Pr}_{2-x} \mathrm{Ce}_{x} \mathrm{CuO}_{4}$
}

\author{
G.B. Martins, D. Rao, J.A. Valdivia, M.A. Pires, G.E. Barberis, and C. Rettori \\ Instituto de Física "Gleb Wataghin," Universidade Estadual de Campinas, 13083-970, Campinas, São Paulo, Brazil \\ P. A. Venegas \\ Departamento de Física, Universidade Estadual Paulista, 17033-360, Bauru, São Paulo, Brazil \\ S. Oseroff \\ San Diego State University, San Diego, California 92182 \\ Z. Fisk \\ Los Alamos National Laboratory, Los Alamos, New Mexico 87545
}

(Received 14 October 1994)

\begin{abstract}
Electron spin resonance (ESR) of $\mathrm{Gd}^{3+}$ and $\mathrm{Er}^{3+}$ in single crystals of $\mathrm{Pr}_{2-x} \mathrm{Ce}_{x} \mathrm{CuO}_{4}(0 \leq x \leq$ 0.15 ) at liquid-helium temperature shows crystal-field (CF) effects corresponding to a $\mathrm{C}_{4 v}$ point symmetry. Upon doping with $\mathrm{Ce}^{4+}$, a reduction of about $23 \%$ in the second-order CF parameter $\left|b_{20}\right|$ is found with no significant change for the other CF spin-Hamiltonian parameters. The resonance lines broaden and present a Dysonian line shape at higher $\mathrm{Ce}^{4+}$ concentration, which is consistent with an increase in the CF inhomogeneity and the metallic character of the compound. Magneticsusceptibility measurements present a small increase in the low-temperature anisotropy upon doping, consistent with a smaller $\left\langle\left|B_{0}^{2}\right|\right\rangle$ crystal-field parameter for $\operatorname{Pr}^{3+}$ in $\operatorname{Pr}_{2-x} \mathrm{Ce}_{x} \mathrm{CuO}_{4}$. The reduction in the CF parameters is tentatively attributed to charge transfer from $\mathrm{Ce}$ atoms to the $\mathrm{CuO}_{2}$ planes. The exchange parameters $j_{\mathrm{Gd}-\mathrm{Pr}}$ and $j_{\mathrm{Pr}-\mathrm{Pr}}$ are estimated from the ESR and susceptibility measurements.
\end{abstract}

\section{INTRODUCTION}

Among the $R_{2} \mathrm{CuO}_{4}$. (2:1:4) compounds, where $R=$ rare-earth, those with $R=\operatorname{Pr}, \mathrm{Nd}$, and $\mathrm{Sm}$ have attracted increased attention since the discovery that superconductivity can be induced in them by substituting Ce for the $R^{1,2}$ This family of superconductors is quite different from the one based on $R=\mathrm{La}$. The La compounds are orthorhombic and superconductivity is achieved by oxidation of the $\mathrm{CuO}_{2}$ planes (hole doping). This is usually achieved by replacing $\mathrm{Sr}^{2+}$ or $\mathrm{Ba}^{2+}$ for $\mathrm{La}^{3+}$ ions or by oxidizing the sample at high temperature and high oxygen pressure, ${ }^{3}$ or even electrochemically. ${ }^{4,5}$ On the other hand, for $R=\mathrm{Pr}, \mathrm{Nd}$, and $\mathrm{Sm}$ the compounds are tetragonal and the substitution of $\mathrm{Ce}^{4+}$ for $R^{3+}$ ions reduces the $\mathrm{CuO}_{2}$ planes (electron doping). It is now known that after thermal treatment in a reducing atmosphere, these cuprates become $n$-type superconductors (negative carriers). ${ }^{2}$ Because of their simpler crystal structure, with planar $\mathrm{CuO}_{2}$ layers and no apical oxygens, the study of these $n$-type superconductors may lead to a better understanding of the fundamental mechanisms of superconductivity in these high-temperature superconductors (HTS).

A common feature of all the 2:1:4 cuprates is that they must be doped in order to achieve superconductivity. It is expected that charge transfer associated with the doping process may locally affect the crystalline electric field (CEF). Various experimental techniques have been used to probe the local CEF: inelastic neutron scattering
(INS), ${ }^{6}$ magnetic susceptibility, ${ }^{7} \mathrm{CF}$ excitations in Raman scattering, ${ }^{8}$ electron spin resonance (ESR), ${ }^{9}$ nuclear quadrupole resonance (NQR) ${ }^{10}$ etc.

Here we present the results of a systematic ESR study at $X$ band of $\sim 0.5 \%$ of $\mathrm{Gd}^{3+}$ and $\mathrm{Er}^{3+}$, in single crystals of $\operatorname{Pr}_{2-x} \mathrm{Ce}_{x} \mathrm{CuO}_{4}(0 \leq x \leq 0.15)$. We also report the results from magnetic susceptibility and ESR experiments on natural impurities of $\mathrm{Gd}^{3+}$ in single crystals of $\mathrm{Pr}_{2} \mathrm{CuO}_{4}$ and $\mathrm{Pr}_{1.85} \mathrm{Ce}_{.15} \mathrm{CuO}_{4}$.

\section{EXPERIMENT}

The samples were grown from nominal stoichiometric mixtures of the corresponding oxides, using $\mathrm{PbO}$ - and CuO-based fluxes in platinum crucibles. ${ }^{11}$ Typical crystal sizes were $3 \times 4 \times 0.3 \mathrm{~mm}^{3}$, with the $c$-axis oriented along the smaller dimension. Our samples were not subjected to reducing thermal treatment, i.e., all our results are for the normal state. The ESR experiments were carried out using a Varian $E$-line spectrometer with a liquid-helium tail Dewar adapted to a room temperature rectangular $T E_{102}$ cavity. The susceptibility measurements were made with a Quantum Design dc SQUID magnetometer.

\section{RESULTS AND ANALYSIS}

Figure 1 shows the observed ESR spectra of $\sim 0.5 \%$ of $\mathrm{Gd}^{3+}$ for several $\operatorname{Pr}_{2-x} \mathrm{Ce}_{x} \mathrm{CuO}_{4}$ crystals at liquid- 
helium temperature, with the magnetic field parallel to the $c$ axis. Figure 2 shows the anisotropy of the spectra for three samples of Fig. 1, when the magnetic field is rotated in the (010) plane. Figure 3 shows the anisotropy of the spectra for the undoped $(x=0)$ and doped $(x=$ $0.15)$ crystals when the magnetic field is rotated perpendicular to the $c$ axis. In every case the anisotropy is well described by the spin Hamiltonian appropriate to $C_{4 v}$ point symmetry: ${ }^{12}$

$$
\begin{aligned}
\hat{H}= & g_{\|} \mu_{B} H_{z} S_{z}+g_{\perp} \mu_{B}\left(H_{x} S_{x}+H_{y} S_{y}\right)+b_{20} O_{20} \\
& +b_{40} O_{40}+b_{44} O_{44}
\end{aligned}
$$

where $g_{\|}$and $g_{\perp}$ are the gyromagnetic factors for the magnetic field parallel and perpendicular to the $c$ axis, respectively. Here $\mu_{B}$ is the Bohr magneton, $O_{n m}$ are Stevens' operators, and $\mathrm{b}_{n m}$ are the corresponding CF parameters. In Eq. (1) we considered spin operators up

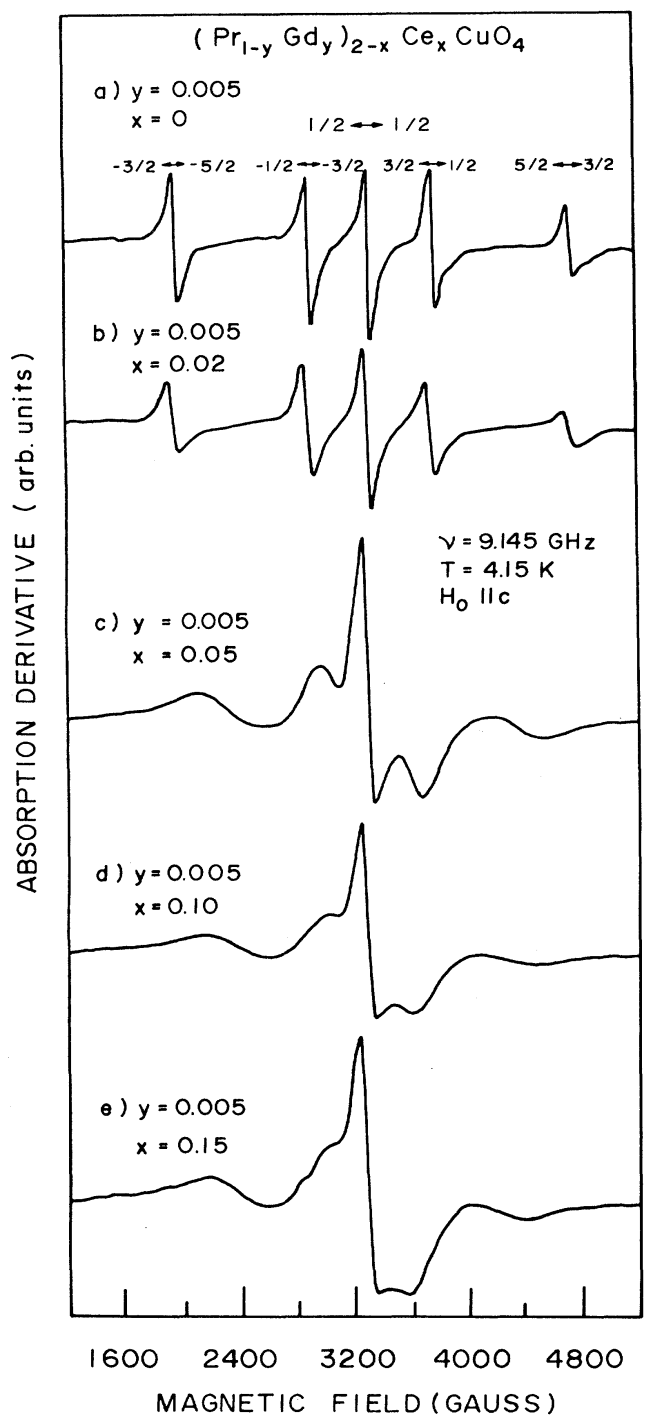

FIG. 1. ESR spectra of $\mathrm{Gd}^{3+}$ in $\operatorname{Pr}_{2-x} \mathrm{Ce}_{x} \mathrm{CuO}_{4}$ for 0 $\leq x \leq 0.15$ and $\vec{H}_{0} \| c$.

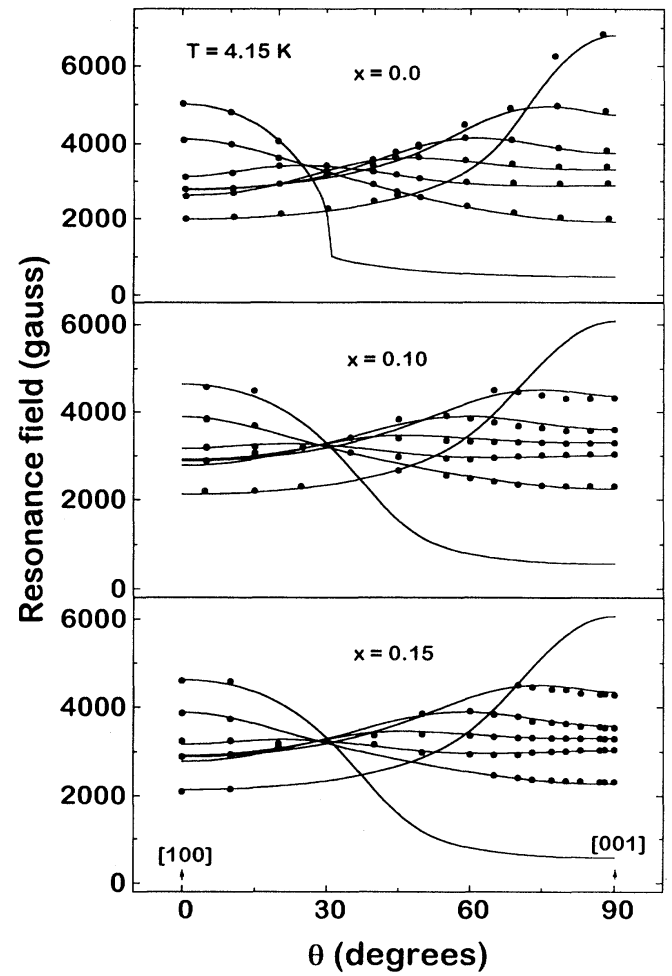

FIG. 2. Angular dependence of the ESR spectra in the (010) plane for $\mathrm{Gd}^{3+}$ in $\operatorname{Pr}_{2-x} \mathrm{Ce}_{x} \mathrm{CuO}_{4}(x=0,0.1,0.15)$. The solid lines are the best fit to Eq. (1) (see text).

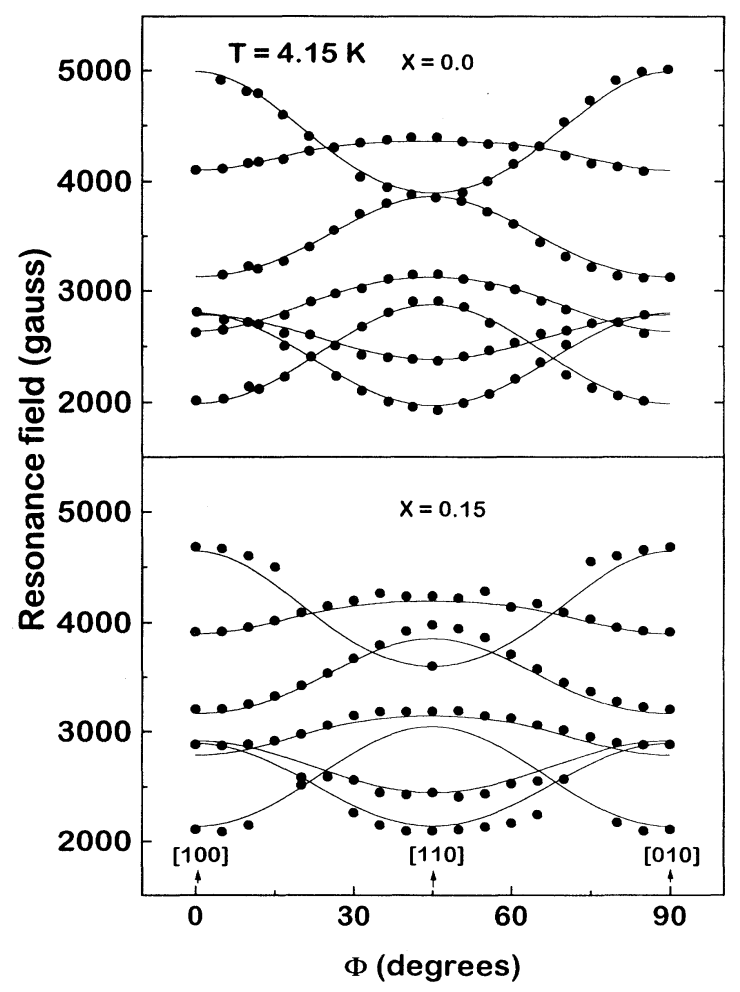

FIG. 3. Angular dependence of the ESR spectra in the (001) plane for $\mathrm{Gd}^{3+}$ in $\mathrm{Pr}_{2-x} \mathrm{Ce}_{x} \mathrm{CuO}_{4}(x=0,0.15)$. The solid lines are the best fit to Eq. (1) (see text). 
to fourth order only, because the values of the sixth order CF parameters are in the range of our experimental error. The solid lines in Figs. 2 and 3 are the best fits of the data to Eq. (1). Since the Zeeman effect is of the same order of magnitude as the crystal-field splitting, the fitting parameters were obtained by complete diagonalization of the spin Hamiltonian in Eq. (1) and a self-consistent calculation of the magnetic field for every transition. Table I shows the fitting parameters obtained at liquid-helium temperature. Since the Zeeman effect is independent of Ce concentration, the $g$ values are not included in Table $\mathrm{I}$. The values obtained at 4.15 K were $g_{\|}=1.985 \pm 0.005$, and $g_{\perp}=2.040 \pm 0.005$. The anisotropy $\left(g_{\perp}-g_{\|}\right)$was found to be smaller $(\sim 0.02)$ at liquid-nitrogen temperature (see below).

Figure 4 shows the simulated spectra corresponding to Fig 1. These spectra were calculated using the parameters given in Table I, the appropriate transition probabilities, and the Boltzmann population factors for each transition. Dysonian line shapes ${ }^{13}$ with increasing metallic character at higher $\mathrm{Ce}^{4+}$ concentration were also used in Fig. 4. We considered linewidths proportional to the matrix elements of the $\mathrm{O}_{20}$ operator for each transition, with increasing values for higher $\mathrm{Ce}^{4+}$ concentrations. The broadening may be attributed to the CF inhomogeneities produced by the doping.

Our data indicate that in addition to a consistently increasing inhomogeneity and metallic character of the samples, associated with the $\mathrm{Ce}^{4+}$ doping process, there is a reduction of about $23 \%$ in the second-order CF parameter $\left|b_{20}\right|$ at the $\mathrm{Gd}^{3+}$ site (see Table I). From a simple point charge model, ${ }^{14}$ and in view of the reduction in the lattice parameters due to the substitution of $\mathrm{Ce}^{4+}$ for $\operatorname{Pr}^{3+}$ ions, ${ }^{15}$ one would expect an increase in $\left|b_{20}\right|$. Instead, our ESR results suggest that the observed reduction in the CF parameter $\left|b_{20}\right|$ may be attributed to charge transfer effects.

In order to see to what extent the change in the second order CF parameter $\left|b_{20}\right|$ of an $S$-state ion $\left(\mathrm{Gd}^{3+}, 4 \mathrm{f}^{7}\right)$ can also be observed in a non- $S$-state ion $\left(\operatorname{Pr}^{3+}, 4 \mathrm{f}^{2}\right),{ }^{16}$ we measured the magnetic susceptibility of two single crystals, $\mathrm{Pr}_{2} \mathrm{CuO}_{4}$ and $\operatorname{Pr}_{1.85} \mathrm{Ce}_{15} \mathrm{CuO}_{4}$. Figure 5 shows the magnetic susceptibility parallel $\left(\chi_{\|}\right)$and perpendicular $\left(\chi_{\perp}\right)$ to the $c$ axis for both crystals. The solid lines in Fig. 5 are the theoretically calculated $\left(\chi_{\|, \perp}\right)$ magnetic susceptibility: ${ }^{17}$

$$
\chi_{\|, \perp}=\frac{\chi_{\|, \perp}^{\mathrm{CF}}}{1-j_{\operatorname{Pr}-\operatorname{Pr}}\left(\frac{g_{J}-1}{g_{J}}\right)^{2} \frac{\chi_{\|, \perp}^{\mathrm{CF}}}{\mu_{B}^{2} N_{0}}}+c N_{0} \frac{\left(g \mu_{B}\right)^{2} S(S+1)}{3 k_{B} T},
$$

TABLE I. Crystal-field parameters for $\mathrm{Gd}^{3+}$ in $\operatorname{Pr}_{2-x} \mathrm{Ce}_{x} \mathrm{CuO}_{4}, b_{n m}\left(10^{-4} \mathrm{~cm}^{-1}\right)$, at $4.15 \mathrm{~K}$.

\begin{tabular}{lccc}
\hline \hline$x$ & $b_{20}$ & $b_{40}$ & $b_{44}{ }^{\mathrm{a}}$ \\
\hline 0 & $-417(5)$ & $-36(2)$ & $42(2)$ \\
0.02 & $-411(5)$ & $-35(2)$ & $40(2)$ \\
0.05 & $-350(10)$ & $-32(3)$ & $40(3)$ \\
0.10 & $-324(20)$ & $-31(4)$ & $40(4)$ \\
0.15 & $-320(20)$ & $-32(4)$ & $40(4)$ \\
\hline \hline
\end{tabular}

${ }^{a}$ See Ref. 25.

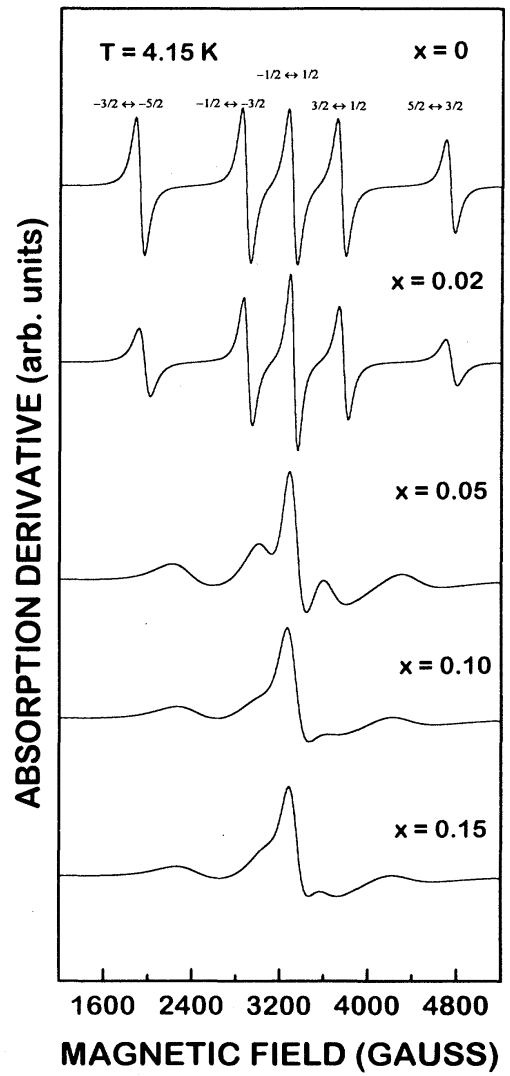

FIG. 4. Simulated ESR spectra (see text) for $\mathrm{Gd}^{3+}$ in $\mathrm{Pr}_{2-x} \mathrm{Ce}_{x} \mathrm{CuO}_{4}$ corresponding to the spectra in Fig. 1.

where $\chi_{\|, \perp}^{\mathrm{CF}}$ is the $\mathrm{Pr}^{3+} \mathrm{CF}$-only magnetic susceptibility, $j_{\mathrm{Pr}-\mathrm{Pr}}$ is the total $\mathrm{Pr}^{3+}-\mathrm{Pr}^{3+}$ exchange interaction, $g_{J}$ the $\operatorname{Pr}^{3+}$ Landé $g$ factor, $N_{0}$ Avogadro's number, and $c$ the concentration of natural impurities relative to $\mathrm{Pr}$, which are assumed to be mainly $\mathrm{Gd}^{3+}(g=2$, $S=7 / 2$ ). In the calculation of $\chi_{\|, \perp}^{\mathrm{CF}}$, all the excited states of $\mathrm{Pr}^{3+}$ were taken into account. We used the intermediate coupling approach ${ }^{18}$ and the CF parameters obtained from Raman experiments. ${ }^{19}$ The host $\mathrm{Pr}^{3+}$ $\mathrm{Pr}^{3+}$ exchange interaction was introduced in Eq. (2) as a molecular field (MF) term. Since we have not seen any splitting of the ESR lines due to antiferromagnetic ordering of the copper ions, ${ }^{20,21}$ we have not taken into consideration any contribution from the copper spins in Eq. (2). For the Ce doped sample, a weighted susceptibility $\chi_{\|, \perp}=\sum_{i} n_{i} \chi_{\|, \perp}^{i}$ was used in order to account for the various $\operatorname{Pr}^{3+}$ sites observed in Raman and INS experiments. ${ }^{6,19}$ The weighting factors $n_{i}$ were obtained from the relative Raman line intensities corresponding to the two sets of sites (sites I, II, IIIa, and sites I, II, IIIb).$^{19}$ The calculated magnetic susceptibility was found to give basically the same result for both sets of sites. Table II displays the set of parameters used in the calculation of $\chi_{\|, \perp}$ for $\operatorname{Pr}_{2} \mathrm{CuO}_{4}$ and $\operatorname{Pr}_{1.85} \mathrm{Ce}_{.15} \mathrm{CuO}_{4}$. The exchange parameter $j_{\mathrm{Pr}-\mathrm{Pr}}$, within the accuracy of the experiment, did not depend on the $\mathrm{Ce}^{4+}$ doping. This is consistent with a very small decrease in the $R$ ordering temperature 


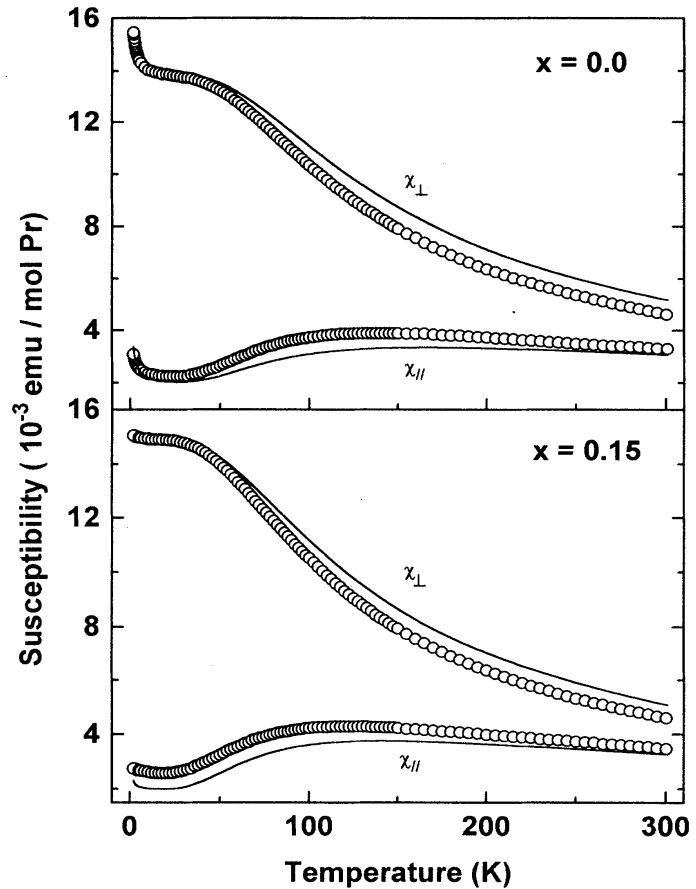

FIG. 5. Temperature dependence of the magnetic susceptibility $(\chi)$ for $\operatorname{Pr}_{2-x} \mathrm{Ce}_{x} \mathrm{CuO}_{4}(x=0,0.15) . \quad \chi_{\|}$and $\chi_{\perp}$ correspond to the magnetic field paralell and perpendicular to the $c$ axis. The applied magnetic field was $10 \mathrm{kOe}$. The solid lines are the calculated susceptibilities using Eq. (2) (see text).

found for the Nd-based (Ref. 22) and Sm-based (Ref. 23) compounds diluted with $\mathrm{Ce}^{4+}$. The temperature dependence of the magnetic susceptibility $\chi_{\|, \perp}$, calculated using the parameters of Table II and presented in Fig. 5, differs from the high-temperature data. We believe that

TABLE II. $\mathrm{Pr}^{3+}$ crystal-field parameters $B_{q}^{k}(\mathrm{meV})$, exchange parameters $j_{R-R}(\mathrm{meV}), \mathrm{Gd}^{3+}$ concentration $c(\mathrm{ppm}$, relative to $\mathrm{Pr}$ ) and weighting factors $n_{i}$ used in the calculated magnetic susceptibility.

\begin{tabular}{rrrrrrr}
\hline \hline \multicolumn{4}{c}{$\mathrm{Pr}_{2} \mathrm{CuO}_{4}$} & \multicolumn{4}{c}{$\mathrm{Pr}_{1.85} \mathrm{Ce}_{15} \mathrm{CuO}_{4}$} \\
\hline$B_{q}^{k}$ & $\mathrm{INS}^{\mathrm{a}}$ & Raman $^{\mathrm{b}}$ & Site I & Site II & Site IIIa & Site IIIb \\
\hline$B_{0}^{2}$ & -28 & -30 & -30 & -17 & -35 & -29 \\
$B_{2}^{2}$ & 0 & 0 & 0 & 0 & 0 & 4 \\
$B_{0}^{4}$ & -301 & -275 & -285 & -297 & -250 & -275 \\
$B_{0}^{6}$ & 26 & 21 & 25 & 41 & 19 & 21 \\
$B_{4}^{4}$ & 228 & 228 & 228 & 228 & 228 & 228 \\
$B_{4}^{6}$ & 224 & 224 & 224 & 224 & 224 & 224 \\
$j_{\operatorname{Pr}-\operatorname{Pr}}$ & $-7(1)$ & \multicolumn{5}{c}{$-7(1)$} \\
$j_{\text {Gd-Pr }}$ & $-0.5(1)$ & & $-0.5(2)$ & \\
$c$ & $\sim 350$ & & \multicolumn{5}{c}{$\sim 70$} & \\
$n_{i}{ }^{\mathrm{c}}$ & & & 0.31 & 0.45 & - & 0.24 \\
$n_{i}{ }^{\mathrm{d}}$ & & & 0.46 & 0.42 & 0.12 & - \\
\hline \hline
\end{tabular}

${ }^{a}$ From Ref. 6.

${ }^{\mathrm{b}}$ From Ref. 19.

${ }^{\mathrm{c}}$ Case 1 from Ref. 19.

${ }^{\mathrm{d}}$ Case 2 from Ref. 19. the difference can be attributed to a temperature dependence of the CF parameters. Some indication for such dependence is suggested by the Raman shift of the 156 $\mathrm{cm}^{-1} \mathrm{CF}$ excitation at high temperatures. ${ }^{8}$

The existence of an exchange interaction between the $R$ 's would certainly introduce a $g$ shift in the $\mathrm{Gd}^{3+}$ resonance. The $g$ shift in the MF approximation can be written $^{17}$

$$
\Delta g_{\|, \perp}=\left[\left(g_{J}-1\right) / g_{J}\right]\left(\frac{\chi_{\|, \perp}}{\mu_{B}^{2} N_{0}}\right) j_{\mathrm{Gd}-\mathrm{Pr}},
$$

where $j_{\mathrm{Gd}-\mathrm{Pr}}$ is the total exchange interaction $\left(j_{\mathrm{Gd}-\mathrm{Pr}}=\right.$ $\sum_{k} j_{\mathrm{Gd}-\mathrm{Pr}}^{k}$ ) between the $\mathrm{Gd}^{3+}$ and the surrounding $\mathrm{Pr}^{3+}$ ions. Equation (3) predicts a smaller $g$-value anisotropy $\left(g_{\|}-g_{\perp}\right)$ at higher temperatures, in agreement with our observations (see above). Using Eq. (3) and the lowtemperature $g$ values, we estimated, within the accuracy of the measurement, $j_{\mathrm{Gd}-\mathrm{Pr}} \cong-0.5 \mathrm{meV}$ in $\mathrm{Pr}_{2} \mathrm{CuO}_{4}$ and $\operatorname{Pr}_{1.85} \mathrm{Ce}_{15} \mathrm{CuO}_{4}$. This value is 1 order of magnitude smaller than $j_{\operatorname{Pr}-\operatorname{Pr}}$ (see Table II), suggesting a trend toward stronger exchange interaction between $R$ 's with larger ionic radius.

Figure 6 shows the ESR spectra of natural impurities of $\mathrm{Gd}^{3+}$ for the same two crystals used in the susceptibility experiment. The presence of natural $\mathrm{Gd}^{3+}$ impurities in the crystals is also evidenced by the small increase in susceptibility at low-temperature shown in Fig. 5. The ESR spectra for these natural impurities are consistent with those for $\mathrm{Gd}^{3+}$-doped crystals given above. We also studied the effect of $\mathrm{Ce}^{4+}$ doping on the ESR spectra of the non-S-state $\mathrm{Er}^{3+}$. Figure 7 shows the ESR spectra of $\mathrm{Er}^{3+}$ in $\operatorname{Pr}_{2-x} \mathrm{Ce}_{x} \mathrm{CuO}_{4}$ for the magnetic field parallel

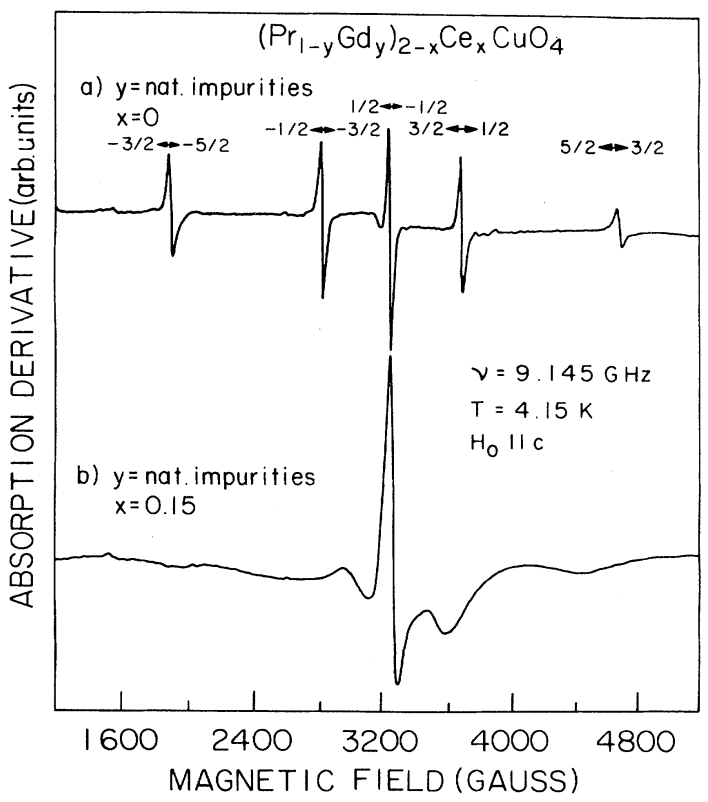

FIG. 6. ESR spectra of $\mathrm{Gd}^{3+}$ natural impurities in $\mathrm{Pr}_{2-x} \mathrm{Ce}_{x} \mathrm{CuO}_{4}(x=0,0.15)$ for $\vec{H}_{0} \| c$. These spectra were obtained from the same samples used in the susceptibility experiments of Fig. 5. 


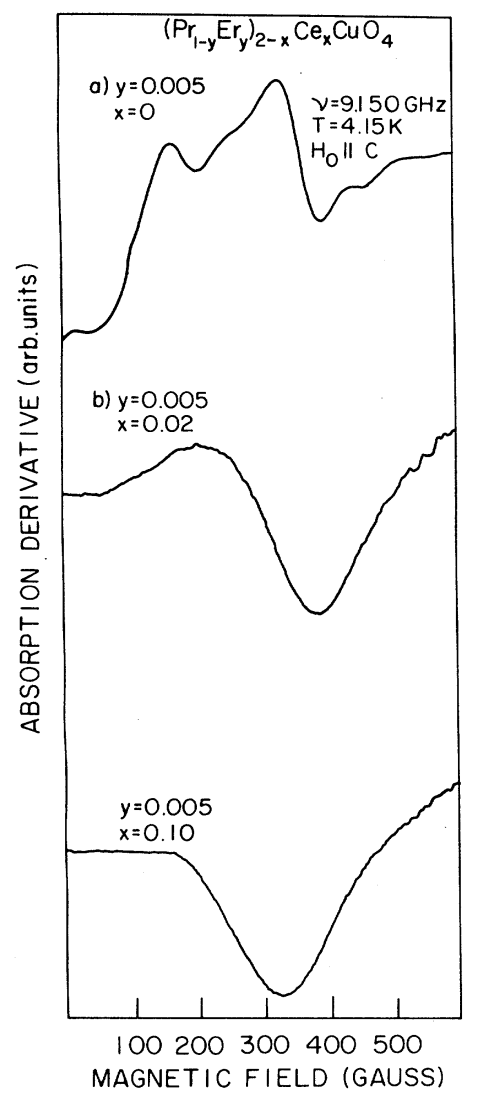

FIG. 7. ESR spectra of $\mathrm{Er}^{3+}$ in $\operatorname{Pr}_{2-x} \mathrm{Ce}_{x} \mathrm{CuO}_{4}(x=0$, $0.02,0.05)$ for $\vec{H}_{0} \| c$.

to the $c$ axis. It is clear from these data that the effect of inhomogeneities is much stronger than for $\mathrm{Gd}^{3+}$. This is expected since $\mathrm{CF}$ effects are first order in non- $S$-states. Figure 8 shows the anisotropy of the $\mathrm{Er}^{3+}$ resonance, which is consistent with the point symmetry at the $R$ site.

\section{CONCLUSIONS}

Our ESR data on $\mathrm{Gd}^{3+}$ and $\mathrm{Er}^{3+}$ in $\mathrm{Pr}_{2-x} \mathrm{Ce}_{x} \mathrm{CuO}_{4}$ show that doping with $\mathrm{Ce}^{4+}$ produces a rather strong local crystal-field perturbation at the lanthanide site. Our most important finding is a reduction of about $23 \%$ in the second-order crystal-field parameter $\left|b_{20}\right|$. This may be attributed to charge transfer in the substitution of $\mathrm{Ce}^{4+}$ for $\mathrm{Pr}^{3+}$ ions. The Ce atoms may act as donor impurities giving electrons to the $\mathrm{CuO}_{2}$ layers, which in turn modify the CEF at the $R$ site.

It is interesting to note that in INS experiments, Boothroyd et $a .^{6}{ }^{6}$ have observed broader peaks for the

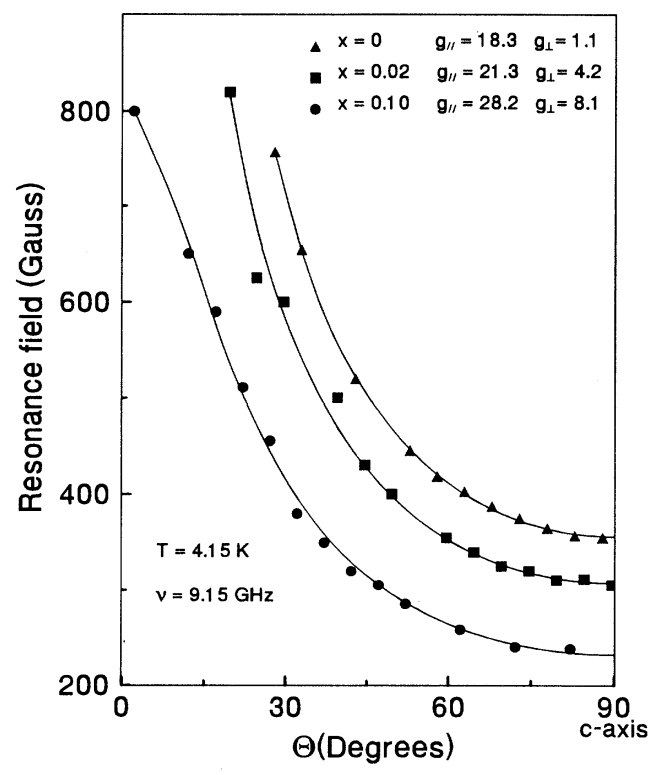

FIG. 8. Angular dependence of the ESR spectra in the (010) plane for $\mathrm{Er}^{3+}$ in $\operatorname{Pr}_{2-x} \mathrm{Ce}_{x} \mathrm{CuO}_{4}(x=0,0.02,0.05)$. Solid lines are the best fit of the resonance field for axial symmetry: $g^{2}(\theta)=g_{\|}^{2} \cos ^{2} \theta+g_{\perp}^{2} \sin ^{2} \theta$.

Ce doped samples. However the peak at $18 \mathrm{meV}$ shows multiple structure with a main feature at $14 \mathrm{meV}$. Moreover, Sanjurjo et $a .^{19}$ also observed recently multiple peak structures in the low-energy part of the Raman CF excitation spectra, similar to that found in the INS experiments. In both experiments, the lower energy peak (14 meV) suggests a reduced $\left|B_{0}^{2}\right| C F$ parameter for $\mathrm{Pr}^{3+}$ in $\mathrm{Pr}_{2-x} \mathrm{Ce}_{x} \mathrm{CuO}_{4}$. The absolute value for a second-order CF parameter weighted among the different sites shown in Table II, $\left\langle B_{0}^{2}\right\rangle=\sum_{i} n_{i} B_{0 i}^{2}$, gives $\left|\left\langle B_{0}^{2}\right\rangle\right| \cong 24-25$ meV. This value is about $21 \%$ smaller than the value for $\mathrm{Pr}_{2} \mathrm{CuO}_{4}$, in good agreement with the ESR result of the present work. The exchange parameter between $\mathrm{Gd}^{3+}$ and $\mathrm{Pr}^{3+}$ is found to be much smaller than that between $\mathrm{Pr}^{3+}$ and $\mathrm{Pr}^{3+}$ (see Table II). This is probably due to the larger ionic radius of $\mathrm{Pr}^{3+}$ ions. We should mention that in recent INS dispersion experiments in $\operatorname{Pr}_{2} \mathrm{CuO}_{4}$, Sumarlin et al. ${ }^{24}$ also interpreted their results in terms of Pr-Pr exchange interaction. As a conclusion, our ESR results suggest that upon doping with Ce there is a charge transfer that modifies the CEF at the lanthanide site.

\section{ACKNOWLEDGMENTS}

This work was supported by Grant No. 91/0573-0 of FAPESP, São Paulo-SP-Brazil, and No. NSF-DMR9117212. 
${ }^{1}$ Y. Tokura, H. Takagi, and S. Uchida, Nature (London) 337, 345 (1989).

${ }^{2}$ H. Takagi, S. Uchida, and Y. Tokura, Phys. Rev. Lett. 62, 1197 (1989).

${ }^{3}$ J.G. Bednorz and K. Müller, Z. Phys. B 64, 189 (1986).

${ }^{4}$ A. Wattiaux, J.C. Park, J.C. Grenier, and M. Pouchard, C.R. Acad. Sci. (Paris) 310, 1047 (1990).

${ }^{5}$ F.C. Chou, D.C. Johnston, S.W. Cheong, and P.C. Canfield, Phys. C 216, 66 (1993).

${ }^{6}$ A.T. Boothroyd, S.M. Doyle, D. McK. Paul, and R. Osborn, Phys. Rev. B 45, 10075 (1992).

${ }^{7}$ M.F. Hundley, J.D. Thompson, S.W. Cheong, Z. Fisk, and S.B. Oseroff, Physica C 158, 102 (1989).

${ }^{8}$ J.A. Sanjurjo, C. Rettori, S. Oseroff, and Z. Fisk, Phys. Rev. B 49, 4391 (1994).

${ }^{9}$ C. Rettori, D. Rao, S. Oseroff, R.D. Zysler, M. Tovar, Z. Fisk, S.W. Cheong, S. Schultz, and D.C. Vier, Phys. Rev. B 44, 826 (1991).

${ }^{10}$ H. Nishihara, H. Yasuoka, T. Shimizu, T. Tsuda, T. Imai, S. Sasaki, S. Kanbe, K. Kishio, K. Kitazawa, and K. Fueki, J. Phys. Soc. Jpn. 56, 4556 (1987).

${ }^{11}$ K.A. Kubat-Martin, Z. Fisk, and R. Ryan, Acta Crystallogr. C 44, 1518 (1988).

${ }^{12} \mathrm{~A}$. Abragam and B. Bleaney, EPR of Transition Ions (Clarendon Press, Oxford, 1970).

13 J.F. Dyson, Phys. Rev. 98, 349 (1955); G. Feher and A.F. Kip, ibid. 98, 337 (1955).

${ }^{14}$ W. Low, Phys. Rev. 109, 265 (1958).

${ }^{15}$ J.M. Tarascon, E. Wang, L.H. Greene, B.G. Bagley, G.W. Hull, S.M. D'Egidio, P.F. Miceli, Z.Z. Wang, T.W. Jing,
J. Clayhold, D. Brawner, and N.P. Ong, Phys. Rev. B 40, 4494 (1989).

${ }^{16}$ D. Davidov, C. Rettori, and D. Shaltiel, Phys. Lett. 50A, 392 (1974); S. Barners, K. Baberschke, and M. Hardiman, Phys. Rev. B 18, 2409 (1978), and references therein.

${ }^{17}$ C. Rettori, D. Davidov, A. Grayevsky, and W.M. Walsh, Phys. Rev. B 11, 4450 (1975).

${ }^{18}$ W.T. Carnall, G.L. Goodman, K. Rajnak, and S. Rana, J. Chem. Phys. 90, 3443 (1989).

19 J.A. Sanjurjo, G.B. Martins, P.G. Pagliuso, E. Granado, I. Torriani, C. Rettori, S. Oseroff, and Z. Fisk, Phys. Rev. B 51, 1185 (1995).

${ }^{20}$ R.D. Zysler, M. Tovar, C. Rettori, D. Rao, H. Shore, S.B. Oseroff, D.C. Vier, S. Schultz, Z. Fisk, and S.W. Cheong, Phys. Rev. B 44, 9467 (1991).

${ }^{21}$ C. Rettori, D. Rao, S.B. Oseroff, G. Amoretti, Z. Fisk, S.W. Cheong, D.C. Vier, S. Schultz, M. Tovar, and R.D. Zysler, Phys. Rev. B 47, 8156 (1993).

22 J.W. Lynn, I.W. Sumarlin, S. Skanthakumar, W-H. Li, R.N. Shelton, J.L. Peng, Z. Fisk, and S.W. Cheong, Phys. Rev. B 41, 2569 (1990).

${ }^{23}$ I.W. Sumarlin, S. Skanthakumar, J.W. Lynn, J. L. Peng, Z.Y. Li, W. Jiang, and R.L. Greene, Phys. Rev. Lett. 68, 2228 (1992).

${ }^{24}$ I.W. Sumarlin, J.W. Lynn, T. Chattopadkyay, S.N. Barilo, and D.I. Zhigunov, Physica C 219, 195 (1994).

${ }^{25}$ In Ref. 9 the value of $b_{44}$ for $\mathrm{Pr}_{2} \mathrm{CuO}_{4}$ is about five times bigger than that shown in Table I. This is because a wrong Stevens' operator coefficient was used in Ref. 9. 